\title{
Dynamic railway network management using switching max-plus-linear models*
}

\author{
T.J.J. van den Boom and B. De Schutter
}

If you want to cite this report, please use the following reference instead: T.J.J. van den Boom and B. De Schutter, "Dynamic railway network management using switching max-plus-linear models," Proceedings of the 11th IFAC Symposium on Control in Transportation Systems, Delft, The Netherlands, pp. 343-348, Aug. 2006.

Delft Center for Systems and Control Delft University of Technology

Mekelweg 2, 2628 CD Delft

The Netherlands

phone: +31-15-278.24.73 (secretary)

URL: https: / / www.dcsc.tudelft.nl

*This report can also be downloaded viahttps://pub. deschutter.info/abs/06_012.html 


\title{
DYNAMIC RAILWAY NETWORK MANAGEMENT USING SWITCHING MAX-PLUS-LINEAR MODELS
}

\author{
Ton J.J. van den Boom ${ }^{*, 1}$ Bart De Schutter*
}

\author{
* Delft University of Technology, \\ Delft Center for Systems and Control, \\ Mekelweg 2, 2628 CD Delft, The Netherlands, \\ $\{t \cdot j \cdot j \cdot$ vandenboom, b. deschutter\}edcsc.tudelft.nI \\ http://dcsc.tudelft.nI
}

\begin{abstract}
In this paper we discuss dynamic traffic management of railway networks. The main aim of the controller is to recover from delays in an optimal way by breaking connections and changing the departure of trains (at a cost). To model the railway system we use a switching max-plus-linear system description. We define the optimal control design problem for the railway network, and we show that solving this problem leads to an integer optimization problem. This problem can be solved with a genetic algorithm or with a mixed integer linear programming algorithm. We also apply the algorithm to a model of the Dutch railway network.
\end{abstract}

Keywords: Railway network management, switching max-plus-linear models, model predictive control

\section{INTRODUCTION}

In recent years a lot of research effort has been oriented towards the design of timetables that are robust against propagation of delays in the network, caused by technical failures, fluctuation of passenger volumes, measures of railway personnel and weather influence (Subiono, 2000; Hansen, 2001; Peeters, 2003; Goverde, 2005). In this paper we concentrate on the operational-level management, and design a feedback controller that takes the most effective actions, based on measurements of the actual train positions. The measures we can take are changing the train speed, breaking train connections, or changing the order of trains.

From (Braker, 1991; Braker, 1993b; de Vries et al., 1998; de Waal et al., 1997; Minciardi et al., 1995) we know that a railway network with rigid connection constraints and a fixed routing schedule can be mod-

\footnotetext{
1 Corresponding author
}

eled using max-plus-linear models. A max-plus-linear model is 'linear' in the max-plus algebra (Baccelli et al., 1992), which has maximization and addition as its basic operations. Max-plus-linear systems can be characterized as discrete event systems in which only synchronization and no concurrency or choice occurs.

Note that in the railway context, synchronization means that some trains should give pre-defined connections to other trains, and a fixed routing means that the order of departure is fixed. However, in the case of large delays, it is sometimes better - from a global performance viewpoint - to break a connection or to reschedule the order of trains, and to let a train depart anyway. In this way we prevent an accumulation of delays in the network. Of course, missed connections should lead to a penalty due to dissatisfied passengers. In (De Schutter and van den Boom, 2001; De Schutter et al., 2002a) we have considered the control of railway networks using breaking connections only as control measure. In (van den Boom and De Schutter, 2004) we have extended the control handles 
and rescheduled the trains by breaking connections as well as changing train order. In this paper we will model a controlled railway system using the switching max-plus-linear system description of (van den Boom and De Schutter, 2006). In this description we use a number of MPL models, each model corresponds to a specific mode, describing the network by a different set of connection and order constraints. We control the system by switching between different modes, allowing us to break train connections and to change the order of trains. In this paper we define a control algorithm to optimize the performance of the network, and we show that the resulting optimization problem can be solved as a mixed integer problem or a mixed integer linear programming problem. Although these problems are in general NP-hard, recently several efficient solvers have become available. The management algorithm will be applied to a simulation model of the Dutch railway network. Computational experiments show that the proposed genetic algorithm approach yields good results.

\section{MODEL}

Consider a railway operations system, which follows a schedule with period $T$. In nominal operation mode, we assume that all the trains follow a pre-scheduled route, with fixed train order and pre-defined connections. If for some reason we have to break connections or change the train order, we will operate in a perturbed mode. With every new schedule we can associate a perturbed mode. First we will discuss the nominal operation.

\subsection{Nominal operation}

Consider a railway operations system which is operating in nominal operation mode.

Each track of the railway network has a number and a train allocated to it. For the sake of simplicity we will say '(virtual) train $j$ ' to denote the (physical) train on track $j$, and 'station $j$ ' to denote the station at the beginning of track $j$ (cf. Figure 1). Let $n$ be the number of 'virtual' tracks in the network. We say virtual to denote that some of the virtual tracks may actually be the same physical track (corresponding to different trains using the same track). This means that the number of tracks is usually smaller than $n$. Let $x_{j}(k)$ be the time instant at which train $j$ departs from station $j$ for the $k$ th time. Let $d_{j}(k)$ be the departure time for this train according to the time schedule. Let $p_{i}(k)$ be the predecessor track of train $i$, and let $\mathcal{C}_{j}(k)$ be the set of trains to which the $k$ th train $j$ gives a connection. Let $\mathcal{F}_{j}(k)$ be the set of trains that move over the same track as train $j$, in the same direction as train $j$, and are scheduled behind train $j$. Let $\mathcal{W}_{j}(k)$ be the set of trains that move over the same track as train $j$, in the opposite direction of

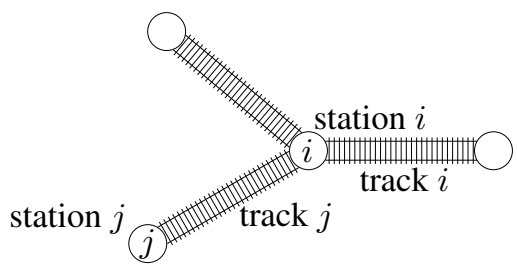

Figure 1. A part of a railway network.

train $j$, and are scheduled behind train $j$. Furthermore, let $a_{j}(k)$ be the traveling time on track $j$, define a minimum connection time $c_{i j}^{\min }(k)$ for passengers to get from train $j$ to train $i$ for each train $j \in \mathcal{C}_{i}(k)$ and define a minimum stopping time $s_{j}^{\min }(k)$ of train $j$ at station $j$ to allow passengers to get off or on the train. Finally, define a minimum separation time $f_{j}^{\min }(k)$ between two different trains moving over the same track and in the same direction as train $j$, and a minimum separation time $w_{j}^{\min }(k)$ between two different trains moving over the same track and in the opposite direction.

Now we have the following constraints for the $k$ th departure time $x_{i}(k)$ of train $i$ :

- Time schedule constraint:

$$
x_{i}(k) \geqslant d_{i}(k) \text {. }
$$

- Continuity constraints: This constraint synchronizes two trains that are 'physically' the same train. For train $j=p_{i}(k)$ we have

$$
x_{i}(k) \geqslant x_{j}\left(k-\delta_{i j}^{*}(k)\right)+a_{j}(k)+s_{j}^{\min }(k)
$$

where $\delta_{i j}^{*}(k)$ is equal to 1 if the $(k-1)$ th train $j$ continues as the $k$ th train $i$, and 0 if the $k$ th train $j$ continues as the $k$ th train $i$ (and if some trips last longer than the twice the cycle time $T$ of the schedule, $\delta_{i j}^{*}(k)$ might be equal to 2 , and so on - see also the example in Section 4). In general, $\delta_{i j}^{*}$ may depend on $k$. However, for the sake of simplicity, we only consider constant $\delta_{i j}^{*}$ 's with a value that is either 0 or 1 in this paper.

- Connection constraints: This constraint synchronizes two trains that have to make a connection. For each train $i \in \mathcal{C}_{j}(k)$ we have

$$
x_{i}(k) \geqslant x_{j}\left(k-\delta_{i j}^{*}\right)+a_{j}(k)+c_{i j}^{\min }(k)
$$

where the role of $\delta_{i j}^{*}$ is similar as for the continuity constraint, so $\delta_{i j}^{*}=1$ if the $(k-1)$ th train $j$ gives a connection to the $k$ th train $i$, and $\delta_{i j}^{*}=0$ if the $k$ th train $j$ gives a connection to the $k$ th train $i$.

- Follow constraints: This constraint synchronizes two subsequent trains on the same track moving in the same direction. For each train $i \in \mathcal{F}_{j}(k)$ we have

$$
x_{i}(k) \geqslant x_{j}\left(k-\delta_{i j}^{*}\right)+f_{j}^{\min }(k)
$$

( $\delta_{i j}^{*}$ is defined similarly as above).

- Wait constraints: This constraint synchronizes two trains on the same track moving in opposite direction. For each train $i \in \mathcal{W}_{j}(k)$ we have

$$
x_{i}(k) \geqslant x_{j}\left(k-\delta_{i j}^{*}\right)+a_{j}(k)+w_{j}^{\min }(k)
$$


( $\delta_{i j}^{*}$ is defined similarly as above).

Since we let a train depart as soon as all connection conditions are satisfied, we have

$$
\begin{aligned}
x_{i}(k) & =\max \left(d_{i}(k),\right. \\
& \left(x_{p_{i}(k)}\left(k-\delta_{i p_{i}(k)}^{*}\right)+a_{p_{i}(k)}(k)+s_{p_{i}(k)}^{\min }(k)\right), \\
& \max _{j \in \mathcal{C}_{i}(k)}\left(x_{j}\left(k-\delta_{i j}^{*}\right)+a_{j}(k)+c_{i j}^{\min }(k)\right), \\
& \max _{l \in \mathcal{F}_{i}(k)}\left(x_{l}\left(k-\delta_{i l}^{*}\right)+f_{l}^{\min }(k)\right), \\
& \left.\max _{m \in \mathcal{W}_{i}(k)}\left(x_{m}\left(k-\delta_{i m}^{*}\right)+a_{m}(k)+w_{m}^{\min }(k)\right)\right)
\end{aligned}
$$

Note that in a undisturbed, well-defined time schedule the term $d_{i}(k)$ in (1) will be the largest. However, if due to unforeseen circumstances (an incident, a late departure, etc.) one of the trains $\left(p_{i}(k), l\right.$ or $m$ ) has a delay the corresponding term can become larger than the others, then train $i$ will depart later than the scheduled departure time $d_{i}(k)$ and will therefore also be delayed. By defining the appropriate matrix $A_{m}^{0}$, $m=0, \ldots, m_{\max }$, (where $m_{\max }=\max \left(\delta_{i j}^{*}\right)$ we can rewrite equation (1) as:

$$
x_{i}(k)=\max \left(d_{i}(k), \max _{j, m}\left(x_{j}(k-m)+\left[A_{m}^{0}\right]_{i, j}\right)\right)
$$

where $\left[A_{m}^{0}\right]_{i, j}$ is the $(i, j)$ th entry of the matrix $A_{m}^{0}$.

Now we introduce some notation from max-plus algebra. Define $\varepsilon=-\infty$ and $\mathbb{R}_{\varepsilon}=\mathbb{R} \cup\{\varepsilon\}$. The maxplus-algebraic addition $(\oplus)$ and multiplication $(\otimes)$ are defined as follows (Baccelli et al., 1992):

$$
x \oplus y=\max (x, y) \quad x \otimes y=x+y
$$

for $x, y \in \mathbb{R}_{\varepsilon}$ and

$$
\begin{aligned}
& {[A \oplus B]_{i j}=a_{i j} \oplus b_{i j}=\max \left(a_{i j}, b_{i j}\right)} \\
& {[A \otimes C]_{i j}=\bigoplus_{k=1}^{n} a_{i k} \otimes c_{k j}=\max _{k=1, \ldots, n}\left(a_{i k}+c_{k j}\right)}
\end{aligned}
$$

for $A, B \in \mathbb{R}_{\varepsilon}^{m \times n}, C \in \mathbb{R}_{\varepsilon}^{n \times p}$. The matrix $\varepsilon$ is the max-plus-algebraic zero matrix: $[\varepsilon]_{i j}=\varepsilon$ for all $i, j$.

In max-plus notation, equation (2) becomes

$$
x_{i}(k)=d_{i}(k) \oplus \bigoplus_{j=1}^{n} \bigoplus_{m=1}^{M} x_{j}(k-m) \otimes\left[A_{m}^{0}\right]_{i, j}
$$

and in matrix-notation we obtain

$$
\begin{aligned}
x(k)= & A_{0}^{0} \otimes x(k) \oplus A_{1}^{0} \otimes x(k-1) \oplus \ldots \\
& \oplus\left[A^{0}\right]_{m_{\max }} \otimes x\left(k-m_{\max }\right) \oplus d(k) \\
= & \left(\bigoplus_{m=0}^{m_{\max }} A_{m}^{0} \otimes x(k-m)\right) \oplus d(k)
\end{aligned}
$$

\subsection{Perturbed operation}

In the nominal operation we have assumed that some trains should give pre-defined connections to other trains, and the order of trains on the same track is fixed. However, if one of the preceding trains has a too large delay, then it is sometimes better - from a global performance viewpoint - to let a connecting train depart anyway or to change the departure order on a specific track. This is done in order to prevent an accumulation of delays in the network. In this paper we will consider the switching between different operation modes, where each mode corresponds to a different set of pre-defined or broken connection and a specific order of train departures. We allow the system to switch between different modes, allowing us to break train connections and to change the order of trains. Note that any broken connection or change of train order leads to a new model, similar to the nominal equation (3), but now with adapted system matrices $\left(A^{\ell}\right)$ for the $\ell$-th model. We have the following system equation for the perturbed operation ${ }^{2}$ :

$$
x(k)=\left(\bigoplus_{m=m_{\min }}^{m_{\max }} A_{m}^{\ell} \otimes x(k-m)\right) \oplus d(k)
$$

\section{THE RAILWAY CONTROL PROBLEM}

\subsection{Timing aspects}

Switching max-plus-linear systems are different from conventional time-driven systems in the sense that the event counter $k$ is not directly related to a specific time. A time instant $t$ in cycle $k$ (so $(k-1) T \leq t<$ $k T)$, some of the components of $x(k)$ may already be known while other components of $x(k)$ may still lie in the future (Recall that $x(k)$ contains the time instants at which the internal activities or processes of the system start for the $k$ th time). Therefore, we will now present a method to address the timing issues in control of switching MPL systems.

We consider the case of full state information ${ }^{3}$, since the components of $x(k)$ correspond to departure times, which are in general easy to measure.

Consider time instant $t$ in cycle $k$, so $(k-1) T \leq$ $t<k T$. We have measurements of departure times $x_{\text {past }}(k)$ and traveling times $a_{\text {past }}(k)$ of trains that have arrived at their destination. Sometimes there is information available about the estimated traveling time for trains that have not yet arrived at their destination at time $t$. With this information we can make an estimation $\hat{a}_{\text {est }}(k \mid t)$ (with the same dimension as $a(k)$ ) of the future traveling times. If no further information is available on a specific traveling time we take the nominal traveling time $\left[\hat{a}_{\mathrm{est}}(k \mid t)\right]_{i}=a_{i, \mathrm{nom}}$.

\footnotetext{
2 Usually $m_{\min }=0$. However, in perturbed operation it may occasionally happen that a delayed train of the $k$ th cycle is rescheduled behind a train in the $(k+1)$ th cycle. In that case we will have $m_{\min }=-1$.

3 Note that measurements of occurrence times of events are in general not as susceptible to noise and measurement errors as measurements of continuous-time signals involving variables such as temperature, speed, pressure, etc.
} 


\subsection{Control problem}

Next we have to define the set $\mathcal{U}(k \mid t)$ of possible future control actions (i.e. breaking connections or changing train order). Certain control actions are not feasible any more (e.g. if a connection has been broken in the past and the connecting train has already departed, it is impossible to 'repair' this connection.). We define the vector $u(k \mid t) \in \mathcal{U}(k \mid t)$, where each element corresponds to a specific control action, so a specific (scheduled) connection or specific (scheduled) train order. We assume $u(k \mid t)$ to be binary, where $u_{i}(k \mid t)=0$ corresponds to the nominal case, and $u_{i}(k \mid t)=1$ to the perturbed case (the connection is broken or the order of two trains is switched, see also Section 3.3).

To select the optimal set of possible future control actions, we define the following optimal control problem at time instant $t((k-1) T \leq t<k T)$ :

$$
\min _{\{u(k \mid t), u(k+1 \mid t), u(k+2 \mid t), \ldots\}} J(k \mid t)
$$

where the performance index $J(k \mid t)$ is given by

$$
J(k, t)=\sum_{j=0}^{\infty} \sum_{i=1}^{n} Q_{i} \hat{e}_{i}(k+j \mid t)+\sum_{\ell=1}^{n_{u}} R_{\ell} u_{\ell}(k+j \mid t)
$$

where $\hat{e}(k+j \mid t)$ is the vector with the expected delays $\left(\hat{e}_{i}(k+j \mid t)=\hat{x}_{i}(k+j \mid t)-d_{i}(k+j) \geq 0\right)$, and $Q, R$ are weighting matrices. The first term of (6) is related to the sum of all predicted delays, and the second term denotes the penalty for all broken connections and switched train orders during cycle $k+j$.

To compute the predictions of $\hat{x}(k+j \mid t)$ we make use of the fact that at time $t$ we have $a_{\text {past }}(k \mid t)$ and $\hat{a}_{\text {est }}(k+j \mid t)$ available and using that we can determine the estimates $\hat{A}^{\ell}(k+j \mid t)$ of all future $A^{\ell}(k+j)$. Now $\hat{x}(k+j \mid t)$ for $j \geq 1$ can be found by successive substitution

$$
\begin{aligned}
\hat{x}(k+j \mid t)= & \hat{A}^{\ell}(k+j-1 \mid t) \otimes \hat{x}(k+j-1 \mid t) \\
& \oplus d(k+j)
\end{aligned}
$$

In principle we have all elements to solve the optimal control problem (5). Note that if the railway network is well-defined and there is some margin in the schedule $^{4}$, there will always be an integer $N$ such that in the nominal case $(u(k+j \mid t)=0$ for all $j \geq 0)$ the delays will have vanished $(\hat{e}(k+j \mid t)=0$ for all $j \geq 0$ ). In the performance index (6) we may then replace the infinite sum by a finite one (with an optional constraint $\hat{e}(k+N \mid t)=0$ ). We now have an integer optimal control problem with $n N$ binary parameters. We can solve this problem efficiently with genetic algorithms (Davis, 1991) or with tabu search (Glover and Laguna, 1997).

\footnotetext{
4 If the max-plus eigenvalue (Braker, 1993a) of the matrix $A^{0}$ is strictly smaller than $T$, there is 'some margin' in the schedule.
}

\subsection{Initial solution}

To find a good initial guess for the integer optimization we first solve an easier problem, in which we structure the input signal. This is done by defining a decision mechanism, where we use thresholds on (expected) delays to decide whether a connection should be broken or train orders should be switched. First consider the case where variable $u_{l}(k)$ is related to the connection of train $j$ to train $i$, with nominal connection constraint

$$
x_{i}(k) \geqslant x_{j}\left(k-\delta_{i j}^{*}\right)+a_{j}(k)+c_{i j}^{\min }(k)
$$

and let $d_{i}(k)>t$. Define $\hat{z}_{j}\left(k-\delta_{i j}^{*} \mid t\right)=\hat{x}_{j}(k-$ $\left.\delta_{i j}^{*} \mid t\right)+\left[\hat{a}_{\mathrm{est}}\right]_{j}(k \mid t)$ as the expected arrival-time of train $j$. Now we choose

$$
\begin{cases}u_{l}(k)=0 & \text { if } \hat{z}_{j}\left(k-\delta_{i j}^{*} \mid t\right)+c_{i j}^{\min }(k)-d_{i}(k) \leq \tau \\ u_{l}(k)=1 & \text { otherwise }\end{cases}
$$

where $\tau$ is a non-negative threshold. Next consider the case where variable $u_{l}(k)$ is related to the order of two trains $j$ and $i$ moving over the same track in the same direction, with nominal following constraint

$$
x_{i}(k) \geqslant x_{j}\left(k-\delta_{i j}^{*}\right)+f_{j}^{\min }(k)
$$

and let $x_{i}(k) \geq t$ (that means that at time $t$ train $x_{i}(k)$ has not departed yet). Now we choose

$$
\begin{cases}u_{l}(k)=0 & \text { if } \hat{x}_{j}\left(k-\delta_{i j}^{*} \mid t\right)+f_{j}^{\min }(k)-d_{i}(k) \leq \phi \\ u_{l}(k)=1 & \text { otherwise, }\end{cases}
$$

where $\phi$ is a non-negative threshold. Finally consider the case where variable $u_{l}(k)$ is related to the order of two trains $j$ and $i$ moving over the same track in the same direction, with nominal waiting constraint

$$
x_{i}(k) \geqslant x_{j}\left(k-\delta_{i j}^{*}\right)+a_{j}(k)
$$

and let $d_{i}(k)>t$. Now we choose

$$
\begin{cases}u_{l}(k)=0 & \text { if } \hat{z}_{j}\left(k-\delta_{i j}^{*} \mid t\right)-d_{i}(k) \leq \omega \\ u_{l}(k)=1 & \text { otherwise, }\end{cases}
$$

where $\hat{z}_{j}\left(k-\delta_{i j}^{*} \mid t\right)$ is the expected arrival-time and $\omega$ is a non-negative threshold. In this structured-input case we end up with the minimization of (6) using the three parameters, giving us a non-linear optimization problem over the variables $(\tau, \phi, \omega)$. In the worked example in the next Section we first optimize over the structured inputs, and use the resulting sequence $u(k+j \mid t)$ as an initial value for the general case, solved with a genetic algorithm.

Remark: The problem above can also be recast as a mixed integer linear programming problem (MILP) using techniques that are similar to the ones used in (Bemporad and Morari, 1999; De Schutter et al., $2002 b$ ). We will now briefly outline the main ideas behind this transformation. Note that the objective function $J$ is linear in $u$ and $\hat{x}$ (via $e$ ). The maxplus equation (7) can be transformed into a system of mixed-integer linear inequalities as follows. Consider an equation of the form $\alpha=\max (\beta, \gamma)$. Hence, we have $\alpha-\beta \geqslant 0$ and $\alpha-\gamma \geqslant 0$. Now assume that 
$\alpha, \beta$ and $\gamma$ are bounded (in practice such bounds are often known due to the operational constraints or due to physical constraints; in our case we could assume that all events take place within the same day). Hence,

$$
\begin{aligned}
& M_{\alpha \beta} \geqslant \alpha-\beta \geqslant 0 \\
& M_{\alpha \gamma} \geqslant \alpha-\gamma \geqslant 0
\end{aligned}
$$

where $M_{\alpha \beta}$ and $M_{\alpha \gamma}$ are upper bounds for $\alpha-\beta$ and $\alpha-\gamma$ respectively. If we now introduce two binary variables $\delta_{1}, \delta_{2} \in\{0,1\}$, then the system of equations

$$
\begin{aligned}
& M_{\alpha \beta} \delta_{1} \geqslant \alpha-\beta \geqslant 0 \\
& M_{\alpha \gamma} \delta_{2} \geqslant \alpha-\gamma \geqslant 0 \\
& \delta_{1}+\delta_{2} \leqslant 1
\end{aligned}
$$

is equivalent to $\alpha=\max (\beta, \gamma)$. Indeed, (10) states that $\delta_{1}=0$ or $\delta_{2}=0$. Hence, by (8)-(9) $\alpha=\beta$ or $\alpha=\gamma$. So $\alpha=\max (\beta, \gamma)$. Furthermore, we could assume that $\hat{A}^{\ell}$ can be written as

$$
\hat{A}^{\ell}(k+j)=\sum_{i=1}^{n_{u}} \hat{A}^{\ell, i}(k+j) u_{i}(k+j \mid k),
$$

which is a linear equation. Now the only nonlinear characteristic problem is the multiplication of entries of $\hat{A}^{\ell}$ - hence, of $u$ by (11) - with entries of $\hat{x}$ due to equation (7). As mentioned in (Bemporad and Morari, 1999) these products can be eliminated as follows: consider the product $\delta y$ of a bounded real variable $y \in[m, M]$ with a binary variable $\delta \in\{0,1\}$. This product is eliminated by introducing an auxiliary real variable $z$, whose value will be equal to $z=\delta y$. More specifically, $z=\delta y$ is equivalent to

$$
\begin{array}{ll}
z \leq M \delta & z \leq y-m(1-\delta) \\
z \geq m \delta & z \geq y-M(1-\delta) .
\end{array}
$$

Hence, we finally end up with an MILP. This problem can then be solved using one of the several existing commercial and free solvers for MILP problems (such as, e.g., CPLEX, Xpress-MP, GLPK, lp_solve, etc. see (Atamtürk and Savelsbergh, 2005; Linderoth and Ralphs, 2004) for an overview).

\section{EXAMPLE: THE DUTCH RAILWAY SYSTEM}

We consider a simulation example of a simplified version of the Dutch railway system (see Figure 2). The Dutch railway system operates a periodic timetable with a cycle time of 1 hour. There are 3 types of trains running in the network: intercity trains, interregional trains and local trains. In this example we only consider the system of intercity and interregional trains, which means that the network consists of 40 stations, 110 tracks, 164 trains, 381 train movements per hour, 271 follow constraints, and 67 connection constraints.

Each train departs as soon as all the relevant connections are guaranteed (except for connections that are broken), the passengers have gotten the opportunity to change over, and the earliest departure time indicated in the timetable has passed.

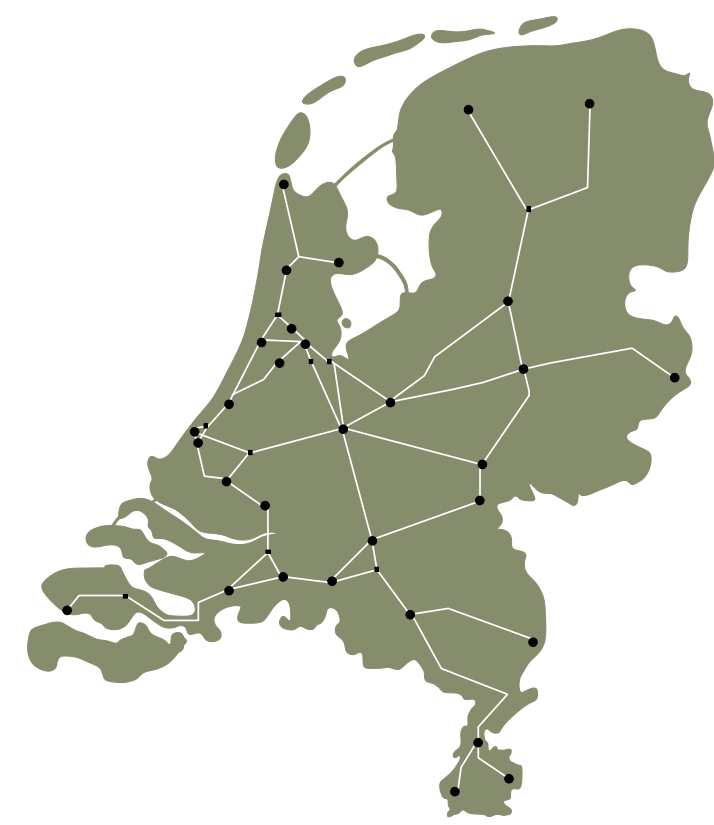

Figure 2. Dutch railway network

We obtain a state space description of the railway system in the form of (2) for nominal operation, and (4) for perturbed operation where $x(k)$ has 381 states. The criterion function is given by (6) where $Q=I$, and $R$ is a diagonal matrix with $R_{\ell, \ell}=5$ when $u_{\ell}$ is related to a connection constraint and $R_{\ell, \ell}=10$ when $u_{\ell}$ is related to a follow constraint. We solve the optimal control problem (5) for the structured input case and the general case (without structuring). In the last optimization we use the result of the structured input as an initial value to start the optimization. We assume the system is at nominal schedule for $k<0$ and we introduce random delays in the running times of 18 trains in the cycles $k=1,2,3$. The maximum delay is $19.9 \mathrm{~min}$, the minimum delay is $0.12 \mathrm{~min}$, and the average delay is $11.9 \mathrm{~min}$. For every cycle $k$ we first optimize the threshold values $(\tau, \phi)$ (there are no single tracks taken into consideration), and compute the corresponding optimal structured input signal $u_{\text {structured }}(k+j \mid t), j \geq 0$. Subsequently we optimize the (unstructured) input signal $u(k+j \mid t)$ with a genetic algorithm, using the earlier computed sequence $u_{\text {structured }}(k+j \mid t)$ as an initial value.

In Figure 3 the maximum delay $e_{\max }(k)=\max (e(k))$ in each cycle $k$ is given for the optimal controlled case, and for the uncontrolled case (so $u(k+j \mid t)=0$ for all $j>0$ ). We see that the delay in the controlled case decays much faster than the uncontrolled case.

\section{DISCUSSION}

We have presented a control design method for a railway network. The control action consists in breaking certain connections or changing the order of departure to prevent delays from accumulating. These control moves can only be done at a certain cost. We have shown that the resulting optimization problem can be 


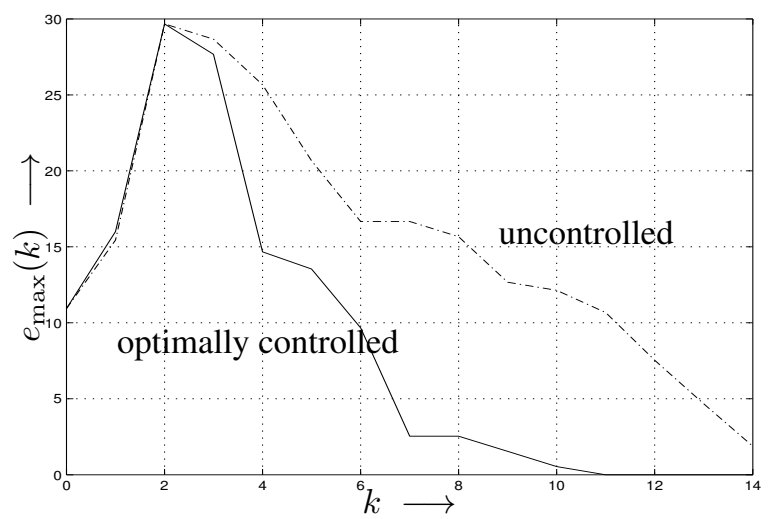

Figure 3. Maximum delay for uncontrolled and optimal controlled railway system

solved using integer optimization methods, for example genetic algorithms or tabu search.

Good initial values for the integer optimization are obtained by first solving a low-dimensional real-valued optimization problem using a structured input sequence. This structured input sequence is based on a decision mechanism, where we use thresholds on (expected) delays to decide whether a connection should be broken or the order of the trains should be switched.

Due to the use of a receding horizon this method can be used in on-line applications and it can deal with (predicted) changes in the system parameters. So if we can predict the delays that will occur due to an incident or to works, then we can include this information when determining the optimal control input for the next cycles of the operation of the network.

\section{Acknowledgments}

Research partially funded by the Dutch Technology Foundation STW project 'Model predictive control for hybrid systems' (DMR.5675), the Transport Research Centre Delft, the European Network of Excellence HYCON (FP6-IST-511368), and by the NWO/STW VIDI project 'Multi-agent control of large-scale hybrid systems' (DWV.6188).

\section{REFERENCES}

Atamtürk, A. and M. W. P. Savelsbergh (2005). Integer-programming software systems. Annals of Operations Research 140(1) 67-124.

Baccelli, F., G. Cohen, G.J. Olsder and J.P. Quadrat (1992). Synchronization and Linearity. John Wiley \& Sons. New York.

Bemporad, A. and M. Morari (1999). Control of systems integrating logic, dynamics, and constraints. Automatica 35(3), 407-427.

Braker, J.G. (1991). Max-algebra modelling and analysis of time-table dependent transportation networks. Proc. 1st European Control Conf. Grenoble, France. pp. 1831-1836.

Braker, J.G. (1993a). Algorithms and Applications in Timed Discrete Event Systems. PhD thesis. Delft Univ. of Technology. Delft, The Netherlands.
Braker, J.G. (1993b). An extended algorithm for performance evaluation of timed event graphs. Proc. 2nd European Control Conf. Groningen, The Netherlands. pp. 524-529.

Davis, L., Ed. (1991). Handbook of Genetic Algorithms. Van Nostrand Reinhold. New York.

De Schutter, B. and T. van den Boom (2001). Model pre-

dictive control for railway networks. Proc. 2001 IEEE/ASME Int. Conf. on Adv. Intelligent Mechatronics (AIM'01). Como, Italy. pp. 105-110.

De Schutter, B., T. van den Boom and A. Hegyi (2002a). A model predictive control approach for recovery from delays in railway systems. Transportation Research Record (1793), 15-20.

De Schutter, B., W.P.M.H. Heemels and A. Bemporad $(2002 b)$. On the equivalence of linear complementarity problems. Operations Research Letters 30(4), 211-222.

de Vries, R., B. De Schutter and B. De Moor (1998). On max-algebraic models for transportation networks. Proc. Int. Workshop on Discrete Event Syst. (WODES'98). Cagliari, Italy. pp. 457-462.

de Waal, P.R., A. Overkamp and J.H. van Schuppen (1997). Control of railway traffic on a single line. Proc. European Control Conf. (ECC'97). Brussels, Belgium, paper 230.

Glover, F. and M. Laguna (1997). Tabu Search. Kluwer Academic Publishers. Boston.

Goverde, R.M.P. (2005). Punctuality of Railway Operations and Timetable Stability Analysis. PhD thesis. Delft University of Technology, TRAIL Thesis Series T2005/10. Delft, The Netherlands.

Hansen, I. (2001). Improving railway punctuality by automatic piloting. Proc. IEEE Intelligent Transportation Systems. Oakland, CA. pp. 792-797.

Linderoth, J. and T. Ralphs (2005). Noncommercial software for mixed-integer linear programming. Optimization Online. 2004/12/1028.

Minciardi, R., M. Paolucci and R. Pesenti (1995). Generating optimal schedules for an underground railway line. Proc. 34th IEEE Conf. Decision and Contr. New Orleans, Louisiana. pp. 4082-4085.

Peeters, L. (2003). Cyclic Railway Timetable Optimization. PhD thesis. Erasmus Univ. Rotterdam, ERIM PhD Series in Management 22. Rotterdam, The Netherlands.

Subiono (2000). On classes of min-max-plus systems and their applications. PhD thesis. Delft Univ. of Technology, TRAIL Thesis Series, T2000/2. Delft, The Netherlands.

van den Boom, T.J.J. and B. De Schutter (2004). Modelling and control of railway networks. Proc. American Control Conf. 2004. Boston, MA. pp. 5728-5733.

van den Boom, T.J.J. and B. De Schutter (2006). Modelling and control of discrete event systems using switching max-plus-linear systems. Accepted for publication in Control Engineering Practice. 